Bangladesh J. Plant Taxon. 19(1): 85-88, 2012 (June)

(C) 2012 Bangladesh Association of Plant Taxonomists

\title{
NEW RECORDS OF EUGLENOPHYCEAE FOR BANGLADESH
}

\author{
Md. Ataul Gani, Md. Almujaddade Alfasane ${ }^{1}$ and Moniruzzaman KhondKer \\ Department of Botany, University of Dhaka, Dhaka 1000, Bangladesh
}

Keywords: Wastewater lagoons; Phytoplankton; Euglena; Lepocinclis; Phacus.

\begin{abstract}
Eight newly recorded species of Euglenophyceae from Bangladesh have been illustrated and described in the present paper. The species are Euglena fundoversata L.P. Johnson, E. korshikovii Gojdics, E. minuta Prescott, E. sacculiformis Schiller, Lepocinclis truncata Da Cunha, Phacus pusillus Lemn., P. strongylus Pochm. and P. wettsteini Drez.
\end{abstract}

\section{Introduction}

A series of papers on the members of Euglenophyceae have been published where 256 taxa have been described and illustrated (Islam and Aziz, 1979; Islam and Khondker, 1981; Islam et al., 1991; Islam and Alfasane, 2002, 2003; Khondker and Alfasane, 2005; Alfasane and Khondker, 2007; Khondker et al., 2008a, b, 2009; Ahmed et al., 2009; Begum, 2009; Begum and Khanam, 2009; Alfasane et al., 2010). During a recent study on planktonic algae of Pagla Sewage Treatment Plant (PSTP) in Narayanganj, Bangladesh, some taxa of the order Euglenales were found to occur, which were not recorded earlier from Bangladesh. In this paper, eight of these taxa are described and illustrated as new records for Bangladesh.

\section{Materials and Methods}

Two lagoons of PSTP at Narayanganj, Dhaka were selected for the present study, namely Lagoon 4 (L-1) and Lagoon 10 (L-2). Detail description of these lagoons of PSTP has been furnished by Gani et al. (2011). A total of 34 phytoplankton samples were collected from October 2009 to July 2010 at fortnight intervals. The samples were collected between 8:00 am and 9:30 am. After collection, the phytoplankton samples were brought to the Phycology, Limnology and Hydrobiology Laboratory of the Department of Botany, University of Dhaka for analysis. Samples were obtained by sieving $100 \mathrm{~L}$ of sub-surface water through a plankton net having a mesh aperture of $20 \mu \mathrm{m}$. The concentrated plankton sample in the bucket of the plankton net were taken in a screw caped glass vial and fixed with Lugol's solution (Gani et al., 2011). A random checking of the sedimented planktonic material was carried out under light microscope with high magnification for identification up to species level. Organisms were photomicrographed with the help of a Nikon Optiphot, UFX-11A microscope fixed with a Nikon FX-35WA camera, Japan.

\section{Taxonomic enumeration}

A total of eight freshwater species of Euglenophyceae have been identified as new reports from Bangladesh. The illustrated taxonomic descriptions of these taxa are given below.

\section{Division: Euglenophyta; Class: Euglenophyceae; Order: Euglenales; Family: Euglenaceae; Genus: Euglena Ehrenberg}

1. Euglena fundoversata L.P. Johnson

(Fig. 1)

(Huber-Pestalozzi 1955, 55, Pl. V, f. 28A; Gojdics 1953, Pl. 2, f. 3a)

\footnotetext{
${ }^{1}$ Corresponding author. Email: mujaddade@yahoo.com
} 
Cell $75 \mu \mathrm{m}$ long, $22 \mu \mathrm{m}$ broad. Cells are relatively large, broad spindle-shaped, end is tapered with a blunt end tip. Body slightly curved. Flagellum about twice as long as body length, flagellum tip is circle. Chromatophores and paramylon numerous.

Collection no. 2 (L-1), 9 Nov 2009.

\section{E. korshikovii Gojdics}

(Fig. 2)

(Gojdics 1953, 52, pl. 19, f. 2a; Syn. E. torta Korshikov)

Cell $33 \mu \mathrm{m}$ long, $19 \mu \mathrm{m}$ broad. Cell undulated, broadly fusiform, tapered. Flagellum equal to body length. Anterior end short, posterior caudus slightly curved. Paramylon numerous, round.

Collection no. 6 (L-1), 15 Jan 2010.

3. E. minuta Prescott

(Fig. 3)

(Huber-Pestalozzi 1955, 118, Pl. XXII, f. 106C; Dillard 2000, Pl.1, f.13)

Cell $14 \mu \mathrm{m}$ long, $5 \mu \mathrm{m}$ broad. Cell small, curved like structure, fusiform to almost pyriform, end tapered short, blunt. Paramylon egg-shaped.

Collection no. 5 (L-2), 30 Dec 2009.

4. E. sacculiformis Schiller

(Fig. 4)

(Huber-Pestalozzi 1955, 114, Pl. XXI, f. 99)

Cell $52 \mu \mathrm{m}$ long, $15 \mu \mathrm{m}$ broad. Cell metabolic, slightly convex to nearly parallel, ends broadly rounded, with short, spine-like apex. Paramylon numerous, small. Huber-Pestalozzi (1955) noted the size of $E$. sacculiformis as $38 \mu \mathrm{m} \times 10 \mu \mathrm{m}$.

Collection no. 6 (L-1), 15 Jan 2010.

\section{Lepocinclis truncata Da Cunha}

\section{Genus: Lepocinclis Perty}

(Huber-Pestalozzi 1955, 146, Pl. XXVIII, f. 138)

Cell $40 \mu \mathrm{m}$ long, $35 \mu \mathrm{m}$ broad. Flagellum half of the body length. Cell pentagonal, anterior end broadly flattened and convex body nature. Two large paramylons.

Collection no. 13 (L-2), 15 May 2010.

Genus: Phacus Dujardin

6. Phacus pusillus Lemn.

(Fig. 6)

(Huber-Pestalozzi 1955, 183, Pl. XXXIV, f. 199, Syn. P. alatus Klebs Partim)

Cell $21 \mu \mathrm{m}$ long, $8 \mu \mathrm{m}$ broad. Cell elongate, median furrow longitudinally arranged. Anterior end slightly blunt, posterior end pointed. Paramylon round.

Collection no. 13 (L-2), 15 May 2010.

7. P. strongylus Pochm.

(Fig. 7)

(Huber-Pestalozzi 1955, 234, Pl. LIV, f. 331; Syn. P. setosa France' Drez.; P. setosa var. polonica Skv. bei Skvortzow; P. setosa var. crenata Skv. bei Skvortzow )

Cell $33 \mu \mathrm{m}$ long, $18 \mu \mathrm{m}$ broad. Cell compressed, straight or slightly curved, dentate in one side view, spiral striation, median furrow dividing into two halves. Caudus long, paramylon numerous.

Collection no. 10 (L-2), 25 Mar 2010. 

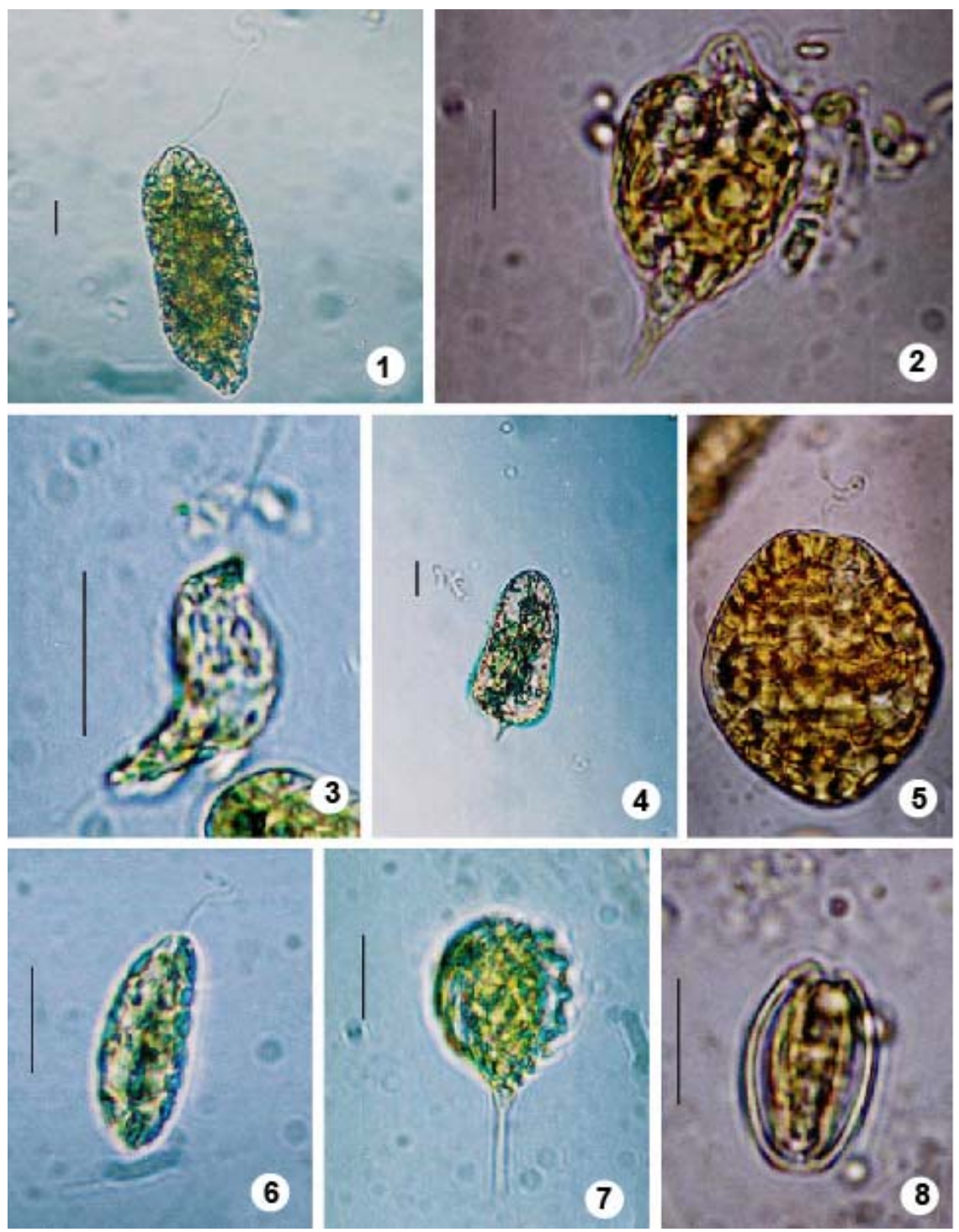

Figs 1-8. 1. Euglena fundoversata L.P. Johnson, 2. E. korshikovii Gojdics, 3. E. minuta Prescott, 4. E. sacculiformis Schiller, 5. Lepocinclis truncata Da Cunha, 6. Phacus pusillus Lemn., 7. P. strongylus Pochm. 8. P. wettsteini Drez. Bar $=10 \mu \mathrm{m}$.

\section{P. wettsteini Drez.}

(Fig. 8)

(Huber-Pestalozzi 1955, 183, Pl. XXXIV, f. 198a)

Cell $15 \mu \mathrm{m}$ long, $7 \mu \mathrm{m}$ broad. Cell ellipsoid to ovoid, anterior front more or less narrows, slightly spreading, with small opening. Posterior end blunt or rounded with bright translucent longitudinal groove, longitudinal striation arranged spirally. Paramylon round.

Collection no. 6 (L-2), 15 Jan 2010. 


\section{References}

Ahmed, Z.U., Khondker, M., Begum, Z.N.T., Hassan, M.A., Kabir, S.M.H., Ahmad, M., Ahmed, A.T.A., Rahman, A.K.A. (eds.) 2009. Encyclopedia of Flora and Fauna of Bangladesh, Vol. 4. Algae, Charophyta - Rhodophyta. Asiatic Society of Bangladesh, Dhaka, 543 pp.

Alfasane, M.A. and Khondker, M. 2007. New records of Phytoplankton for Bangladesh: Phacus, Lepocinclis and Pteromonas Bangladesh J. Plant Taxon. 14(2): 167-169.

Alfasane, M.A., Islam, M.S. and Khondker, M. 2010. Some freshwater phytoplankton as new reports from Bangladesh. Bangladesh J. Plant Taxon. 17(1): 87-92.

Begum, Z.N.T. 2009. A taxonomic account on the phytoplankton of a pond receiving textile industrial effluents. II. Euglenophyceae and Bacillariophyceae. Bangladesh J. Plant Taxon. 16(1): 9-19.

Begum, Z.N.T. and Khanam, D. 2009. Physicochemical asspects and phytoplankton of the river Shitalakhya receiving pharmaceutical effluents. Bangladesh J. Bot. 38(1): 77-85.

Dillard, G.E. 2000. Freshwater algae of the Southeastern United States. Part 7. Pigmented Euglenophyceae. Bibl. Phycol. Bd. 106. J. Cramer, Berlin, Stuttgart, 135 pp. + 20 pls.

Gani, M.A., Alfasane, M.A. and Khondker, M. 2011. Limnology of wastewater treatment lagoons at Pagla, Narayanganj. Bangladesh J. Bot. 40(1): 35-40.

Gojdics, M. 1953. The Genus Euglena. The Univ. Wisconsin Press, Madison, 268 pp. + 39 pls.

Huber-Pestalozzi, G.H. 1955. Das Phytoplankton des Süßswassers: Systematik und Biologie. Euglenophyceen E. Schweizerb. Verlagsb. (Nägele u. Obermiller), Stuttgart, Germany, pp. 606 + Pls. 1114.

Islam, A.K.M. Nurul and Aziz, A. 1979. Algal flora of Moheshkhali Island, Bangladesh. Dhaka Univ. Stud. B 27(2): 105-122.

Islam, A.K.M. Nurul and Alfasane, M.A. 2002. Euglenophyceae from Barisal district, Bangladesh: I. Genus Phacus. Bangladesh J. Plant Taxon. 9(2): 3-18.

Islam, A.K.M. Nurul and Alfasane, M.A. 2003. Euglenophyceae from Barisal district, Bangladesh: II. Lepocinclis, Strombomonas and Trachelomonas. Bangladesh J. Plant Taxon. 10(1): 15-26.

Islam, A.K.M. Nurul and Khondker, M. 1981. Euglenophyta of Bangladesh. I. Genus Trachelomonas Ehr. Int. Revue ges. Hydrobiol. 66(1): 109-125.

Islam, A.K.M. Nurul, Khondker, M. and Haque, S. 1991. Euglenoid algae of four polluted ponds in and around Dhaka city. Bangladesh J. Bot. 20(1): 7-15.

Khondker, M. and Alfasane, M.A. 2005. Euglenamorpha hegneri Wenrich (Euglenaceae): A rare euglenoid from Bangladesh. Bangladesh J. Bot. 34(1): 41-43.

Khondker, M., Bhuiyan, R.A., Yeasmin, J., Alam, M., Sack, R.B., Huq, A. and Colwell, R.R. 2008a. New records of phytoplankton for Bangladesh. 5. Euglena, Euglenocapsa. Bangladesh J. Plant Taxon. 15(1): 39-46.

Khondker, M., Bhuiyan, R.A., Yeasmin, J., Alam, M., Sack, R.B., Huq, A. and Colwell, R.R. 2008b. New records of phytoplankton for Bangladesh. 8. Trachelomonas Ehr. (Euglenophyceae). Bangladesh J. Bot. 37(2): 133-139.

Khondker, M., Bhuiyan, R.A., Yeasmin, J., Alam, M., Sack, R.B., Huq, A. and Colwell, R.R. 2009. New records of phytoplankton for Bangladesh. 9. some rare and a new species. Bangladesh J. Plant Taxon. 16(1): 1-8. 